\title{
XMM-Newton detection of Nova Muscae 1991 in quiescence
}

\author{
F. K. Sutaria ${ }^{1}$, U. Kolb ${ }^{1}$, P. Charles ${ }^{2}$, J. P. Osborne ${ }^{3}$, E. Kuulkers ${ }^{4, \star}$, J. Casares ${ }^{5}$, E. T. Harlaftis ${ }^{6}$, T. Shahbaz ${ }^{7}$, \\ M. Still ${ }^{8}$, and P. Wheatley ${ }^{3}$ \\ 1 The Open University, Milton Keynes, UK \\ 2 University of Southampton, UK \\ 3 X-ray Astronomy Group, Dept of Physics and Astronomy, University of Leicester, University Rd, Leicester LE1 7RH, UK \\ 4 SRON, Utrecht, The Netherlands \\ 5 IAC, La Laguna, Tenerife, Spain \\ ${ }^{6}$ Institute of Astronomy and Astrophysics, National Observatory of Athens, PO Box 20048, Athens 11810, Greece \\ 7 Dept. of Astrophysics, Oxford University, UK \\ 8 NASA/GSFC, Maryland, USA
}

Received 19 March 2002 / Accepted 4 June 2002

\begin{abstract}
The soft X-ray transient GU Mus has been detected by XMM-Newton in the quiescent state. The source is very faint, with a $0.5-10.0 \mathrm{keV}$ unabsorbed flux of $\simeq 1.1 \times 10^{-14} \mathrm{ergs} \mathrm{cm}^{-2} \mathrm{~s}^{-1}$. The spectra is well fit by an absorbed powerlaw with a photon index of $\alpha=1.6 \pm 0.4$, close to the value seen when the source was in the low/hard state in Aug. 1991. From our observed luminosity, it seems unlikely that the quiescent state emission is dominated by coronal X-rays from the secondary. The flux also appears to be in agreement with the ADAF model of BH-transients in quiescence.
\end{abstract}

Key words. X-rays: binaries - X-rays: individuals: GU Mus - black hole physics - accretion, accretion disks

\section{Introduction}

Soft X-ray transients (SXTs) are compact binaries where a neutron star (NS) or black hole (BH) primary accretes from a Roche-lobe filling donor star. While there exists unique observational signatures for the identification of a neutron star in these systems, e.g. through $\mathrm{X}$-ray bursts which are thought to be due to thermonuclear burning of superheated accreted matter on the surface of a neutron star, there is no similar identifier for an event horizon - the unique characteristic of a BH-system. A knowledge of the mass function of the system (from optical observations of the companion), can at most give a lower limit to the mass of the compact object. In the case of stellar mass BHs, this is usually not very definitive, given the present uncertainty on the limiting mass of neutron stars.

The spectra of SXTs in outburst appear to be dominated by a radiatively efficient, optically thick, geometrically thin, accretion disc in a hot, high viscosity state. However, spectra of BH-SXTs in quiescence do not appear to be a simple accretion disc phenomenon alone (e.g. Lasota 2001). A simple, two component model for SXTs in quiescence consists of an outer, thin, accretion disc, which serves as a reservoir for the accumulated mass. An inner, hot, advection-dominated accretion flow (ADAF) transports energy from the inner edge of the accretion

Send offprint requests to: F. K. Sutaria,

e-mail: F.K.Sutaria@open.ac.uk

^ Present address: ESA-ESTEC, Noordwijk, The Netherlands. disc to the compact object (Menou et al. 1999). In the case of NS-SXTs, this would heat up the surface of the NS (without thermonuclear runaway) causing it to radiate, or, in the case of BH systems, carry the energy, along with matter, out of sight beyond the event horizon. Thus the quiescent state spectra of SXTs would yield information on the nature of the compact object. Hence, in recent years the quiescent properties of SXTs and their white dwarf analogues (dwarf novae) have taken centre stage in the quest to prove the existence of black holes by detecting the event horizon, and in the search for the holy grail of accretion disc theory, the viscosity mechanism (Garcia et al. 2001; Narayan et al. 2001).

The two-component model discussed above is not without inconsistencies. Inclusion of viscous heating of electrons, along with that of protons, accounting for convectively unstable flows, and magneto-rotational instability in 3-D numerical computations of ADAFs now show that ADAFs develop strong outflows (Hawley et al. 2001). Also, the large mass flow inherent in ADAF models predicts a much larger $L_{\text {quiesc }}$ in NS systems than observed. However, all ADAF models consistently predict that BH-SXTs should have a much lower quiescent flux (by a factor of $\simeq 100$ ) relative to the NS SXTs.

Prompted by the apparent failure of ADAF for NS-SXTs, alternative explanations for the quiescent emission have been put forward, including coronal emission from companion stars (e.g. Bildsten \& Rutledge 2000; but these exceed the existing 
quiescent BH SXT detections), and emission from the neutron star surface that was heated in earlier outbursts (Brown et al. 1999).

So far, quiescent state detections, or upper limits, exist for 6 NS-SXTs and 8 BH-SXTs. The quiescent luminosities of these systems in the $0.5-10.0 \mathrm{keV}$ range (Table 2, Narayan et al. 2001), normalised to $10^{38} \mathrm{ergs} \mathrm{s}^{-1}$ are plotted as a function of the orbital period $P_{\text {orb }}$ in Fig. 1. Systems with similar orbital periods are expected to be at the same point in their evolutionary history, and hence expected to have similar accretion rates $\dot{M}$ (however, see Sect. 4 ). In accordance with the ADAF models, the BH-SXTs do appear to be fainter than the NS SXTs. We discuss the cause of this scatter in Sect. 4. We had designed our observation of the BH-SXT GU Mus in order to (a) investigate the ADAF prediction of the relative faintness of quiescent BH systems over NS systems with similar orbital period and (b) to obtain a potential spectral signature for the existence of an event horizon - i.e., the search for the indisputable evidence of a black hole. GU Mus is an ideal candidate for testing these hypotheses because no X-ray activity has been detected from it since the last outburst in Jan. 1991. We discuss the outburst properties, the present observation and our results in the following sections.

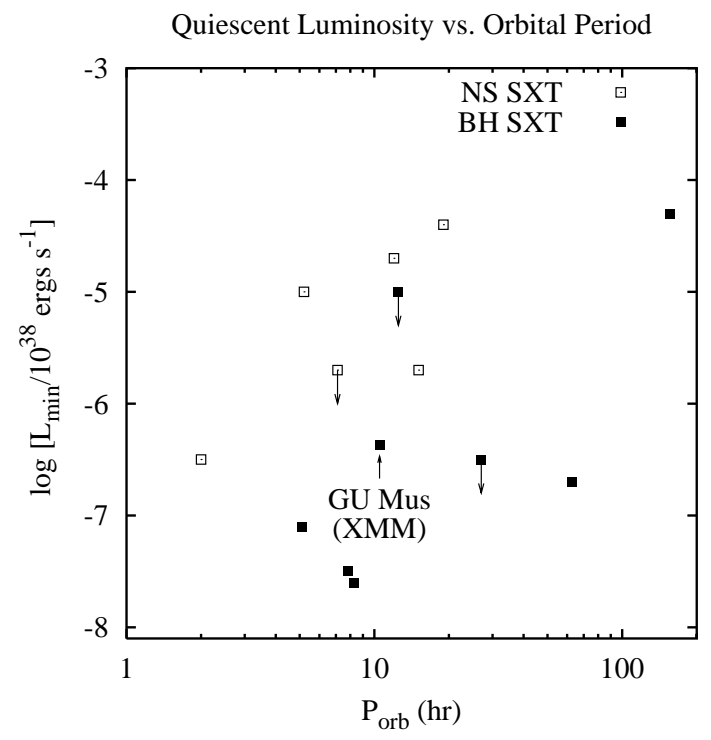

Fig. 1. Quiescent luminosities of BH- and NS- SXTs plotted against their orbital periods. The data is taken from Table 1 of Narayan et al. (2001). All luminosities are in the 0.5 to $10 \mathrm{keV}$ band.

\section{Earlier observations of SXT GU Mus (Nova Muscae 1991)}

In X-rays, GU Mus (also known as GS 1124-68, Nova Mus, Nova Muscae 1991) was initially detected as a bright SXT in outburst by GRANAT-WATCH and the Ginga-ASM detectors, between Jan. 9 to 11, 1991 (Lund et al. 1991; Makino 1991), and by the ROSAT-All Sky Survey during Jan. 24/25 1991. The combined high/soft state ROSAT and Ginga spectrum was fitted by a two component model, consisting of a multitemperature disk blackbody (Mitsuda et al. 1984), with a maximal disk temperature of $0.96 \mathrm{keV}$ plus a hard powerlaw component with a photon index of $\simeq 2.45$ (Greiner et al. 1994). Subsequent photometric observations of the optical counterpart during April 92 to July 94 revealed a periodicity of $\sim 10.38 \mathrm{hr}$ in this source (Orosz et al. 1996). From optical spectroscopic measurements in quiescence (Orosz et al. 1996, and references therein), the mass function for the system was measured at $f(M)=3.01 \pm 0.15 M_{\odot}$, and limits on the inclination angle were set at $54^{\circ} \leq i \leq 65^{\circ}$. The mass of the compact object and its companion are $3.86 \pm 0.19\left(\sin ^{-3} i\right) M_{\odot}$, and $0.51 \pm 0.06\left(\sin ^{-3} i\right) M_{\odot}$ respectively. Infrared observations (Gelino et al. 2001) set narrower limits on the inclination angle $i=54^{\circ} \pm 1.5^{\circ}$, and restrict the mass of the BH to $6.95 \pm 0.6 M_{\odot}$.

Distance estimates for GU Mus range from $2.8 \mathrm{kpc}$ to $\geq 5.5 \mathrm{kpc}$, depending on the mass of the compact object, the spectral type of the companion, and the accretion model. Optical photometric observations of the companion, revealed it to be a main sequence dwarf star with spectral type K3-5 V, thus implying a distance of $5.5 \pm 1 \mathrm{kpc}$. Infrared observations in 1995 (Shahbaz et al. 1995) of GU Mus give a distance limits of $2.8 \mathrm{kpc}$ to $4.0 \mathrm{kpc}$. Finally, combined X-ray Ginga and ROSAT observations of GU Mus in outburst yielded an X-ray column density $N_{\mathrm{H}}=2.6 \times 10^{21} \mathrm{~cm}^{-2}$, assuming a thin disc model for the spectra. This was consistent with the extinction obtained from both IUE $\left(E_{B-V} \simeq 0.2-0.3\right)$, and HST observations $\left(E_{B-V}=0.287 \pm 0.004\right.$, Cheng et al. 1992), and is larger than the expected galactic reddening in this direction, suggesting that the GU Mus transient is located behind the galactic disc (Greiner et al. 1994). Because the estimate of distance from $\mathrm{X}$-ray observations is highly model dependent, we use here the "optical value" of $d=5.5 \mathrm{kpc}$ as our reference distance.

Prior to 1991, there were no outbursts detected from this source (Greiner et al. 1994). Following the 1991 outburst, the source luminosity showed an exponential decline typical of BH-systems, with a characteristic timescale of 21.9 days. Though there was a second luminosity increase $\sim 80$ days after the outburst, and again at $\sim 200$ days, the source showed an overall steady decline in luminosity, and it fell below the Ginga/LAC detection limits $(0.3 \mathrm{mCrab}) 282$ days after the outburst (Ebisawa et al. 1994). It was not detected in the ROSAT (0.3-2.4 keV) band $\sim 410$ days after outburst, and the source flux had fallen below $\simeq 5.0 \times 10^{-14} \mathrm{ergs} \mathrm{cm}^{-2} \mathrm{~s}$. It has remained in the quiescent state ever since, with no significant detection reported until now.

\section{The XMM-Newton observation}

GU Mus was observed by XMM-Newton detectors on Feb. 2425,2001 , with an exposure time of $\sim 34100 \mathrm{~s}$. We used pipeline processed data for our analysis, because a comparison of the calibration files used in the pipeline processing with those used at the time of analysis suggested that no significant improvements would have resulted with any recalibration of the data. The events in the EPIC-MOS1, EPIC-MOS2 and EPIC-PN datasets were filtered using the SAS-xmmselect task, using the event selection criteria recommended in the "Users Guide to the XMM-Newton Science Analysis System” (2001). The total 
counts in the three EPIC CCDs from GU Mus was $~ 100$. Both EPIC-MOS and EPIC-PN observations were carried out in the thin filter mode.

Among the various sources detected on the EPIC CCDs, we identified GU Mus from its optical coordinates (RA = $11^{\mathrm{h}} 26^{\mathrm{m}} 26^{\mathrm{s}} .65$ and Dec $=-68^{\circ} 40^{\prime} 32^{\prime \prime} \cdot 2$, J2000) (Della Valle et al. 1991). From a time series analysis of the entire EPIC-PN, MOS1 and MOS2 data, we find that there are times of large, random fluctuation over the entire field of view of both EPIC$\mathrm{PN}$ and MOS CCDs. This high background rate over almost $50 \%$ of the exposure time was possibly due to detection of soft protons, or other high energy particles by the EPIC-CCDs. By restricting our analysis to the intervals of low background, our effective exposure time was reduced to $\sim 15800 \mathrm{~s}$.

The data was binned and analysed using the XSPEC (version 11.1) package. The response and auxilary (rmf- and arf- ) files were generated for each CCD using the SAS tasks "rmfgen" and "arfgen" respectively. We extracted the background spectrum from a region of the chip which was free of any significant sources, and which was on same section of the CCD as the source. For EPIC-PN, in the 0.3 to $14 \mathrm{keV}$ range, the countrate for the background spectra was $9.4( \pm 2.4) \times 10^{-4} \mathrm{~s}^{-1}$ and the background subtracted countrate in the source region was $1.64( \pm 0.45) \times 10^{-3} \mathrm{~s}^{-1}$. Though faint, the source stands well above the statistical fluctuation of the background. The background subtracted countrates in EPIC-MOS1 $(0.3-12 \mathrm{keV})$ was $0.72( \pm 0.44) \times 10^{-3} \mathrm{~s}^{-1}$ and in EPIC-MOS2 (0.3-12 kev) it was $0.77( \pm 0.57) \times 10^{-3} \mathrm{~s}^{-1}$. The count rate in the MOS1 and MOS2 detectors is too low to give useful spectra even after binning, so since the MOS1 and MOS2 have similar response functions, their spectra were grouped together and analysed. The EPIC-PN spectra are analysed separately.

For both EPIC-PN and EPIC-MOS, because of the very low number of counts, a meaningful fit was only possible if the column density was kept fixed. We chose $N_{\mathrm{H}}=2.6 \times$ $10^{21} \mathrm{~cm}^{-2}$ - as obtained from fits to ROSAT and Ginga data (see above). The EPIC-PN data were binned so as to produce at least 8 counts/bin, and the MOS1 and MOS2 data were binned to at least 5 counts/bin. Since $\chi^{2}$ fitting requires about 30 counts/bin to be meaningful, we used the Cash-statistic (XSPEC C-statistic, see Cash 1979) here instead, which puts better limits on the fitting parameters (Table 1). XSPEC version 11.1, used here, allows C-statistic fits on data with the background spectra read in. The C-statistic is defined as the logarithm of the probability that the number of counts in a given phase bin is actually reproduced by the fitted model. Since Cstat does not provide a goodness of fit, for the purpose of comparison, we computed both the goodness of fit from MonteCarlo probability calculations (Col. 5, Table 1), as well as the $\chi^{2}$ statistic. The "goodness of fit" should hover around $50 \%$ if the observed data was produced by the fitted model. The chistatistic was calculated for each fit using the parameters obtained from C-statistic fitting and quoted in the Table. Figure 2 shows the EPIC-PN spectrum fitted using an absorbed powerlaw model and $\mathrm{C}$-statistic. The $\chi^{2}$-deviation displayed in the figure was calculated using the $\mathrm{C}$-stat fitted parameters.

In addition to the absorbed power-law model, we have also fitted our data to the absorbed bremsstrahlung, Raymond-Smith

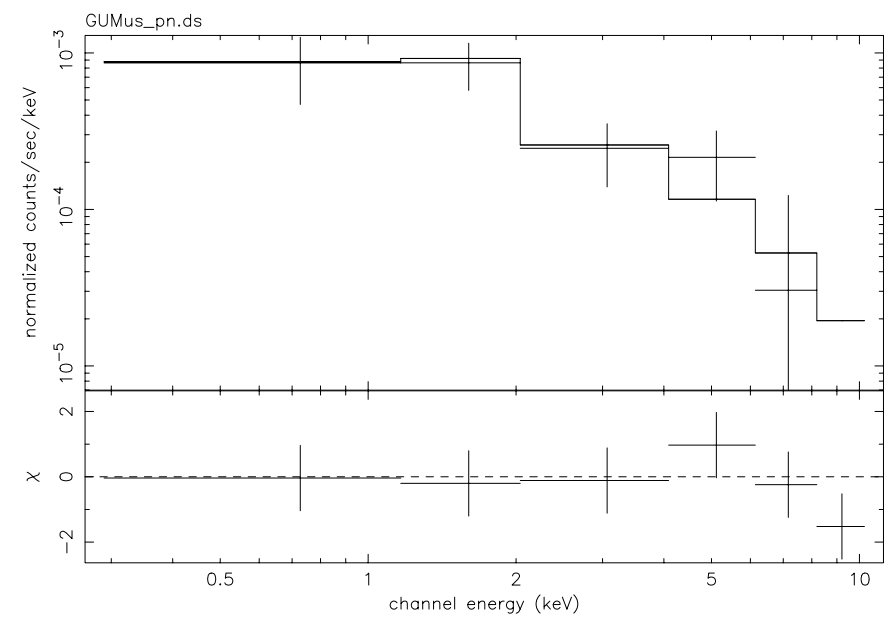

Fig. 2. EPIC-PN spectrum and the fitted model (see Sect. 3) of GU Mus in quiescence. The absorbed powerlaw model is fitted here, with $\log N_{\mathrm{H}}$ fixed at 21.4. The residuals are plotted in the lower panel.

(solar abundance) and black-body models. The EPIC-PN spectra were fitted over the $0.2-12 \mathrm{keV}$ range, and the results for each model are presented in Table 1. In order to facilitate comparison with the Chandra observations of the spectra of other BH-SXT (Kong et al. 2002), we quote the (absorbed) flux in the $0.3-7 \mathrm{keV}$ range as well as in the $0.3-12 \mathrm{keV}$ range in Table 1. The fits to the EPIC-PN spectra suggests that while the $\chi_{v}^{2} /$ d.o.f. are comparable for all models used, the blackbody model can at least be counted out, because of the very low value of absorption. Similarly, the bremsstrahlung and the coronal emission model (Raymond-Smith) also constrain the parameter values very poorly, so we are inclined to treat the absorbed powerlaw as the model that best describes the (admittedly sparse) data. Using the $90 \%$ confidence limits on the powerlaw fit with constant $\alpha$ (see Fig. 3), the unabsorbed, $0.5-10 \mathrm{keV}$ flux is $1.1_{-0.4}^{+1.1} \times 10^{-14} \mathrm{ergs} \mathrm{cm}^{-2} \mathrm{~s}^{-1}$. The energy range $0.5-10.0 \mathrm{keV}$ is being used to allow comparison with the earlier (mainly Chandra) observations plotted in Fig. 1. Since the distance to GU Mus is uncertain, to estimate the luminosity we use a reference distance $d=5.5 \mathrm{kpc}$, as obtained from optical observations (Orosz et al. 1996). Thus the unabsorbed EPIC-PN flux quoted above implies a luminosity of $4.0 \times 10^{31}(d / 5.5 \mathrm{kpc})^{2} \mathrm{ergs} \mathrm{s}^{-1}$ in the $0.5-10 \mathrm{keV}$ range.

The MOS1 and MOS2 data were fitted simultaneously, allowing only the relative normalisations to vary. Fitting the $0.3-$ $10 \mathrm{keV}$ spectra, and using an absorbed powerlaw model, we find that C-statistic fit parameters are reasonable only if we keep $N_{\mathrm{H}}$ constant. The best fit photon index was $\alpha=1.43 \pm$ 0.52 , for $\chi_{v}^{2} /$ d.o.f. $=1.15 / 7$. The absorbed $0.5-10.0 \mathrm{keV}$ flux is $1.8 \times 10^{-14}$ and $1.2 \times 10^{-14} \mathrm{ergs} \mathrm{cm}^{-2} \mathrm{~s}^{-1}$ in EPIC-MOS1 and MOS2 respectively. The other three models quoted in Table 1 do not fit the data well, resulting in unrealistic and badly constrained values of the temperature parameter in each model, and hence are not reported here.

The source was too weak to be detected in the RGS1 and RGS2 spectrometers, nor was it detected in the UVM2 and UVW1 filters by the optical monitor. For an exposure time of $2860 \mathrm{~s}$, the limiting count rate for detection in the 
Table 1. Best fit spectral parameters for EPIC-PN. $k T$ represents the plasma temperature or the black body temperature, depending on the model. All uncertainties are quoted in $90 \%$ confidence limit. The absrobed flux is quoted here.

\begin{tabular}{cccccccc}
\hline \hline Model & $N_{\mathrm{H}}$ & $\alpha$ & $k T$ & $\mathrm{CASH}$ & $\chi_{v}^{2} /$ d.o.f. & $\left.f\right|_{0.3-7.0}$ & $\left.f\right|_{0.3-12.0}$ \\
\hline & $10^{21}$ & & & $\mathrm{M}-\mathrm{C}$ & & $\times 10^{-14}$ & $\times 10^{-14}$ \\
& $\mathrm{~cm}^{-2}$ & & $\mathrm{keV}$ & Prob. & & $\mathrm{ergs} \mathrm{cm}^{-2} \mathrm{~s}^{-1}$ & $\mathrm{ergs} \mathrm{cm}^{-2} \mathrm{~s}^{-1}$ \\
\hline Power-law & $2.3_{-2.3}^{+8.9}$ & $1.6_{-0.9}^{+1.6}$ & - & $44.8 \%$ & $1.12 / 3$ & 0.90 & 1.31 \\
& 2.6 (const.) & $1.6_{-0.6}^{+0.8}$ & - & $44.9 \%$ & $0.85 / 4$ & 0.93 & 1.34 \\
Bremsstrahlung & $1.9_{-1.9}^{+0.1}$ & - & $9.7_{-8.2}^{+190.2}$ & $38.4 \%$ & $1.05 / 3$ & 0.91 & 1.12 \\
& 2.6 (const.) & - & $7.4_{-5.6}^{+192.6}$ & $39.9 \%$ & $0.82 / 4$ & 0.88 & 1.12 \\
Raymond-Smith & $1.8_{-1.8}^{+7.4}$ & - & $11.1_{-9.5}^{+52.9}$ & $45.8 \%$ & $1.11 / 3$ & 0.96 & 1.30 \\
& 2.6 (const.) & - & $6.3_{-4.5}^{+57.7}$ & $43.8 \%$ & $0.87 / 4$ & 0.97 & 1.20 \\
Black body & $1.6 \times 10_{-1.6 \times 10^{-4}}^{+3.9}$ & - & $0.88_{-0.40}^{+0.48}$ & $49.5 \%$ & $1.35 / 3$ & 0.87 & 0.91 \\
& 2.6 (const.) & - & $0.83_{-0.46}^{+0.52}$ & $67 \%$ & $1.5 / 4$ & 0.84 & 0.87 \\
\hline
\end{tabular}

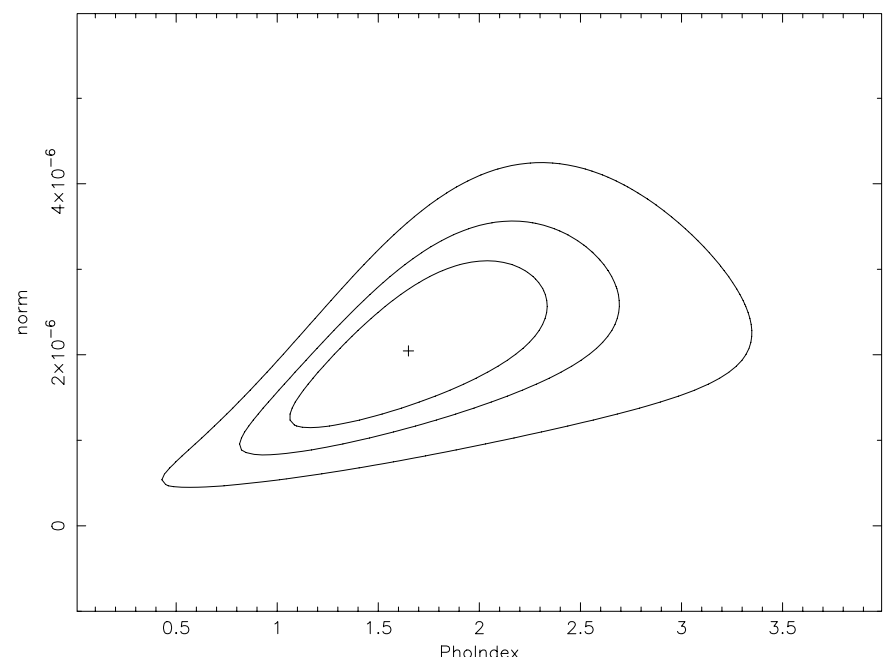

Fig. 3. The contours plotted above are for $68.3 \%, 90 \%$ and $99 \%$ confidence limits on the values of the photon index $\alpha$ and powerlaw normalisation norm, assuming that $N_{\mathrm{H}}=2.6 \times 10^{21} \mathrm{~cm}^{-2}$.

UVW1 filter was $2.4 \times 10^{-2} \mathrm{~s}^{-1}$, and in the UVM2 filter, it was $1.36 \times 10^{-2} \mathrm{~s}^{-1}$.

\section{Discussion}

Our observation had been planned with the intention of using the spectral characteristics of GU Mus to provide an observational discriminant between NS- and BH-SXTs. However, we find that, while we have a clear detection of GU Mus in the quiescent state, at $\sim 100$ counts, the spectra have insufficient counts to constrain well even the simple absorbed power-law model. By fixing the column density at $N_{\mathrm{H}}=0.26 \times 10^{22} \mathrm{~cm}^{-2}$, the fit to the EPIC-PN spectra constrains the photon index to $\alpha=1.6 \pm 0.4$. This appears to be consistent with both the outburst and post-outburst observations, where the photon index of the hard component decreased from its high/soft state value of 2.2-2.7 (Jan. to Apr. 1991) to its low/hard value of $\sim 1.6$ in Sep. 1991 (Ebisawa et al. 1994). Besides GU Mus, only 5 other BH-SXTs have been actually detected in quiescence (for Chandra observations, see Kong et al. 2001; Garcia et al. 2001) and these luminosities have been plotted in Fig. 1, as a function of orbital period $P_{\text {orb }}$. In addition, upper limits for the BH SXTs $4 \mathrm{U} 1543-47\left(P_{\text {orb }}=27 \mathrm{hr}\right.$, Orosoz et al. 1998) and H1705-250 $\left(P_{\text {orb }}=12.7 \mathrm{hr}\right.$, Narayan et al. 1997$)$ have also been plotted. The luminosities for the Chandra observations included in Fig. 1, were calculated (Narayan et al. 2001 and references therein) in the energy range $0.5-10.0 \mathrm{keV}$, by fitting to a power-law spectrum with photon index $\alpha \sim 2.0$ and column densities consistent with the optical extinction.

The evaluation of the intrinsic X-ray luminosity of GU Mus in quiescence is uncertain, chiefly because of the poor constraints on the distance. In Fig. 1, we have plotted the value of $\log L_{\text {quies }} / 10^{38}=-6.40$ for GU Mus, using our reference value of $5.5 \mathrm{kpc}$. In terms of the Eddington luminosity, using an inclination of $i=60^{\circ}, L_{0.5-10.0} / L_{\mathrm{Edd}}=9.25 \times 10^{-8}(d / 5.5 \mathrm{kpc})^{2}$. We plot the luminosities in Fig. 1 as normalised to a factor of $10^{38} \mathrm{ergs} \mathrm{s}^{-1}$, instead of the ratio $L_{\text {quiesc }} / L_{\text {Edd }}$, as preferred by others (e.g. Garcia et al. 2001), because the Eddington luminosity $L_{\text {Edd }}$ will be $\sim 10$ times larger in $\mathrm{BH}$ systems than in NS systems, thus leading to an artificial separation between the BH and NS systems. A natural separation can be expected in compact binaries with $P \leq 12 \mathrm{hrs}$ when the accretion rate $\dot{M}$ is driven by orbital angular momentum losses $\dot{J}$. If these are dominated by magnetic braking, $\dot{J}$ is the same for identical donor stars, so that $\dot{M}$ is $\propto 1 / J$ i.e. roughly to $1 / M_{\text {primary }}$ (e.g. King et al. 1997). Hence $\dot{M}$ in BH systems would be expected to be smaller than in NS systems. Normalisation relative to $L_{\mathrm{Edd}}$ is reasonable only if the event were driven by gravitational radiation, since, in that case, $\dot{M}$ would scale with $M$. However, the truth may be more complicated. Some short-period NS systems may be remnants of a thermal-timescale mass transfer phase (King et al. 2001), and at least one short-period BH system (XTE J1118+480) shows evidence nuclear-processed material (Haswell et al. 2001), a clear sign of an evolved donor. The secondary in the BH-SXT GS $2000+25$, too, has been found to be an evolved donor (Harlaftis et al. 1996). All this suggests a greater spread of $\dot{M}$ at a given $P_{\text {orb }}$.

An alternative explanation for SXT quiescent X-ray emission was given by Bildsten \& Rutlidge (2000), who suggested that it arose from the corona of the (necessarily) rapidly rotating companion star. In order to investigate this possibility, we fitted our data to the Raymond-Smith model for a 
coronal plasma, assuming solar abundances. If the emission is indeed coronal in origin, rather than an ADAF in the inner disk region, then the temperature of the plasma should be $\leq 1.4 \mathrm{keV}$ (Dempsey et al. 1993), and the ratio of the soft $\mathrm{X}$-ray to bolometric luminosities be $L_{\mathrm{SX}} / L_{\mathrm{bol}}<10^{-3}$. While our fits constrain the temperature too poorly to compare, we do note that the absorbed flux in the $0.4-2.4 \mathrm{keV}$ range is $3.3 \times 10^{-15} \mathrm{ergs} \mathrm{cm}^{-2} \mathrm{~s}^{-1},\left(\right.$ EPIC-PN spectra with constant $N_{\mathrm{H}}$ ) which implies, $L_{\mathrm{SX}} / L_{\mathrm{bol}}>8.96 \times 10^{-3}$. In order to calculate $L_{\text {bol }}$, we used $V=20.5$ for GU Mus, with a $50 \%$ contribution from the accretion disk (Orsoz et al. 1996), resulting in $V_{\text {secondary }}=21.25$. The other parameters were $E(B-V)=0.3$ (Della Valle et al. 1991), $A_{V}=3.1 E(B-V)$ and distance $d=5.5 \mathrm{kpc}$. Thus, based on luminosity estimates alone, it maybe unlikely that the quiescent state X-ray emission originates from the companion star's corona. We also note, however, that of all the other BH-SXTs observed in quiescence so far (GRO J0422+32, A0620-00, XTE J1550-564, V404 Cyg, GRO J1655-40 and GS 2000+25, Kong et al. 2000), the stellar corona appears to contribute a major portion of the soft X-ray luminosity only in GS $2000+25$, although as yet no spectra exists for the quiescent state of this source.

\section{References}

Cheng, F. H., Horne, K., Panagia, N., et al. 1992, ApJ, 397, 664 Bildsten, L., \& Rutledge, R. E. 2000, ApJ, 541, 908 Brown, E. F., Bildsten, L., \& Rutledge, R. E. 1998, ApJ, 504, L95 Cash, W. 1979, ApJ, 228, 939

Della Valle, M., Jarvis, B. J., \& West, R. M. 1991, Nature, 353, 50
Dempsey, R. C., Linsky, J. L., Schmitt, J. H. M. M., \& Fleming, T. A. 1993, ApJ, 413, 333

Ebisawa, K., Ogawa, M., Aoki, T., et al. 1994, PASJ, 46, 375

Garcia, M. R., McClintock, J. E., Aoki, T., et al. 2001, ApJ, 553, 47

Gelino, D. M., Harrison, T. E., \& McNamara, B. J. 2001, AJ, 122, 971

Greiner, J., Hasinger, G., Molendi, S., \& Ebisawa, K. 1994, A\&A, 285,509

Harlaftis, E. T., Horne, K., \& Filippenko, A. V. 1996, PASP, 108, 762

Haswell, C. A., Hynes, R. I., King, A. R., \& Schenker, K. 2002, MNRAS, accepted

Hawley, J. F., Balbus, S. A., \& Stone, J. M. 2001, ApJ, 554, 49

Lasota, J.-P. 2001, New Astron. Rev., 45, 449

Lund, N., \& Brandt, S. 1991, IAU Circ., 5161

King, A. R., Kolb, U., \& Szuszkiewicz, E. 1997, ApJ, 488, 89

King, A. R., Schenker, K., Kolb, U., \& Davies, M. B. 2001, MNRAS, 321, 327

Kong, A. K. H., McClintock, J. E., Garcia, M. R., Murray, S. S., \& Barret, D. 2002, ApJ, accepted [astro-ph/0111134]

Mitsuda, K., Inoue, H., Koyama, K., et al. 1984, PASJ, 36, 741

Makino, F. 1991, IAU Circ., 5161

Menou, K., Eisen, A., Narayan, R., et al. 1999, ApJ, 520, 276

Narayan, R., Garcia, M. R., \& McClintock, J. E. 1997, ApJ, 478, L79

Narayan, R., Garcia, M. R., \& McClintock, J. E. 2001 [astro-ph/0107387]

Orosz, J. A., Bailyn, C. D., McClintock, J. E., \& Remillard, R. A. 1996, ApJ, 468, 380

Orosoz, J. A., Jain, R. K., Bailyn, C. D., McClintock, J. E., \& Remillard, R. A. 1998, ApJ, 499, 375

$\mathrm{Ph}$. Gondoin 2001, Users guide to the XMM-Newton Science Analysis System, XMM-Newton Science Operations center, Feb. 23

Shahbaz, T., Naylor, T., \& Charles, P. A. 1997, MNRAS, 285, 607 